\title{
Erratum to: Strategic delegation in consumer cooperatives under mixed oligopoly
}

\author{
Michael Kopel · Marco A. Marini
}

Published online: 3 January 2014

(C) Springer-Verlag Wien 2013

\section{Erratum to: J Econ}

\section{DOI 10.1007/s00712-013-0384-y}

Due to a mistake on the part of Springer, the presentation of Table 1 was wrong in the initially published version. The correct version of Table 1 is given below:

Table 1 The first stage subgame with the corresponding payoffs for the two firms

\begin{tabular}{|c|c|c|c|}
\hline & & \multicolumn{2}{|l|}{ Coop } \\
\hline & & $N D$ & $D$ \\
\hline \multirow[t]{2}{*}{$P M F$} & $N D$ & $\pi_{1}^{N D N D}=\frac{A(1-\beta)}{4(1+\beta)}, V^{N D N D}=\frac{A(5+3 \beta)}{8(1+\beta)}$ & $\pi_{1}^{N D D}=\frac{A(1-\beta)\left(2-\beta^{2}\right)^{2}}{(1+\beta)\left(4-\beta^{2}\right)^{2}}, V^{N D D}=\frac{A\left(5+3 \beta-\beta^{2}-\beta^{3}\right)}{2\left(4+4 \beta-\beta^{2}-\beta^{3}\right)}$ \\
\hline & $D$ & $\pi_{1}^{D N D}=\frac{A(1-\beta)}{4(1+\beta)}, V^{D N D}=\frac{A(5+3 \beta)}{8(1+\beta)}$ & $\pi_{1}^{D D}=\frac{A(1-\beta)\left(2-\beta^{2}\right)}{8(1+\beta)}, V^{D D}=\frac{A\left(20+12 \beta+\beta^{3}-\beta^{2}\right)}{32(1+\beta)}$ \\
\hline
\end{tabular}

The online version of the original article can be found under doi:10.1007/s00712-013-0384-y.

\section{Kopel}

Department of Organization and Economics of Institutions, University of Graz, Graz, Austria e-mail: michael.kopel@uni-graz.at

\section{A. Marini ( $\square)$}

Department of Computer, Control and Management Engineering, Sapienza Università di Roma, Via Ariosto, 25, 00185 Rome, Italy

e-mail: marini@dis.uniroma1.it

M. A. Marini

CREI, Rome, Italy 